\title{
Ubiquitin ligases join the field of dietary restriction in C.elegans
}

\author{
Mark Lucanic and Pankaj Kapahi
}

\author{
Buck Institute for Age Research, Novato, CA 94945, USA
}

Running title: Ubiquitin ligases and dietary restriction

Key words: C elegans, dietary restriction, ubiquitin ligases

Correspondence: Pankaj Kapahi, PhD, Buck Institute for Age Research, 8001 Redwood Blvd., Novato, CA 94945, USA

Received: 08/18/09; accepted: 09/01/09; published on line: 09/03/09

E-mail: pkapahi@buckinstitute.org

Copyright: (C) 2009 Lucanic and Kapahi. This is an open-access article distributed under the terms of the Creative Commons Attribution License, which permits unrestricted use, distribution, and reproduction in any medium, provided the original author and source are credited

Dietary restriction (DR) has been shown to robustly modulate organismal lifespan in multiple species. The powerful genetic tools in yeast, worms and flies hold great promise in unraveling the secrets of the molecular mechanisms of this process that have been elusive for more than seven decades, since DR was first described to extend lifespan in mice. In a recent study, Carrano and colleagues report the discovery that an E2/E3 ubiquitin ligase set are required for the lifespan extending effects of DR in C.elegans [1].

The authors utilize C. elegans to look at E3 ubiquitin ligases of the conserved HECT (Homologous to E6-AP Carboxyl Terminus) family known to be important in tumorigenesis in mammals [2]. C. elegans contains a single ortholog of the WW class of HECT E3s known as WWP-1. Mutant analysis revealed that the C. elegans gene is required for embryonic development but that weak mutants or naïve adults exposed to $w w p-1$ dsRNA have wildtype lifespan under normal culture conditions but are sensitive to heat and oxidative stress. Stress response genes are often involved in modulating normal lifespan in a positive manner. Consistent with this notion the authors find that overexpression of wwp-1 leads to a lengthened lifespan in otherwise wildtype animals. As these results implicate WWP-1 as having both a stress resistance and pro-longevity function the authors next test whether the gene contributes to other known longevity pathways.

Insulin/IGF signaling, mitochondrial function and nutrients control distinct aspects of C. elegans lifespan. To test whether WWP-1 functions in any of these longe- vity pathways, the authors examine mutant combinations with essential components of these pathways. They find that a loss of wwp-1 in long lived insulin/IGF and mitochondrial mutants results in a modest shortening of their extended lifespan. However, the lifespan extension in eat-2 mutants, which eat less due to reduced pharyngeal pumping rate and are therefore under DR, is abrogated. They also convincingly show that WWP-1 is involved in DR by using a method that utilizes bacterial dilution to impart lifespan extension. One of the dramatic findings was that the 3 fold extension of lifespan achieved by reducing food concentration in liquid culture is almost entirely suppressed by a loss of $w w p-1$. These experiments genetically implicate WWP-1 as being a key player in the DR pathways of longevity.

WWP-1 is orthologous to mammalian E3 ubiquitin ligases and the authors show biochemically, that WWP1 also has E3 activity in vitro. Disruption of a key residue in the catalytic domain abolished both the in vitro activity and its ability to rescue the DR phenotype. As part of the ubiquitin targeting process E3s function in a cascade that has immediately upstream of it an E2 ubiquitin conjugating enzyme, WWP-1 had previously been found to interact with a specific E2 ubiquitin conjugating enzyme known as UBC-18 [3]. The authors find from their in vitro assay that most of the functional E3 activity of WWP-1 is dependent on the presence of UBC-18. Furthermore, they show that the two proteins (UBC-18 and WWP-1) interact in a GST pull down assay. Finally the authors show that $u b c-18$ behaves 
genetically similar to $w w p-1$ in that it also is required for DR induced lifespan extension.

An understanding of the molecular pathways that mediate the protective effects of DR is one of the most important questions in biomedicine. Previous studies in C. elegans have identified novel genetic pathways that determine the beneficial effects of DR $[4,5,6,7,8]$. Key regulators of DR-dependent lifespan extension include transcription factors and environmental sensors such as PHA-4, SKN-1, HSF-1, and AMPK, which were identified by different dietary regimens that restrict nutrient intake. TOR (Target of Rapamycin) is also emerging as a key regulator in mediating the effects of DR and is conserved across species. It is has been shown to modulate lifespan extension in flies [9], yeast [10], worms [11] and most recently mice [12]. The signaling pathways that mediate the effects of DR are complex and impinge on a variety of cellular targets. For example, the TOR pathway was recently shown to modulate the effects of DR in C. elegans through HIF-1 acting downstream of S6K to modulate DR-dependent lifespan extension via the IRE-1 ER stress pathway [13]. It is now becoming apparent that the maximal lifespan extension by DR is not achieved by regulating a single genetic pathway but that multiple pathways act together to mediate the lifespan effect of DR. Future research examining how the E2/E3 ligase set identified by Carrano and colleagues interacts with other pathways of DR in C. elegans will be of great interest. Importantly the E2/E3 ligase set likely functions to degrade or modulate a target protein(s) whose nonubiquitinated function shortens lifespan in stressful conditions such as low nutrient availability and high temperature. Identification of the upstream signaling cascades that activate this E2/E3 ligase set and their specific targets should yield a better understanding of how animals modulate their lifespan based on environmental conditions such as nutrient availability.

\section{CONFLICT OF INTERESTS STATEMENT}

The authors of this manuscript have no conflict of interests to declare.

\section{REFERENCES}

1. Carrano AC, Liu Z, Dillin A, Hunter T. A conserved ubiquitination pathway determines longevity in response to diet restriction. Nature. 2009; 460:396-399.

2. Bernassola F, Karin M, Ciechanover A, Melino G. The HECT family of E3 ubiquitin ligases: multiple players in cancer development. Cancer Cell. 2008; 14:10-21.
3. Qiu X, Fay DS. ARI-1, an RBR family ubiquitin-ligase, functions with UBC-18 to regulate pharyngeal development in C. elegans. Dev Biol. 2006; 291:239-252.

4. Panowski SH, Wolff S, Aguilaniu H, Durieux J, Dillin A. PHA4/Foxa mediates diet-restriction-induced longevity of $C$. elegans. Nature. 2007; 447:550-555.

5. Greer EL, Dowlatshahi D, Banko MR, Villen J, Hoang K, et al. An AMPK-FOXO pathway mediates longevity induced by a novel method of dietary restriction in C. elegans. Curr Biol. 2007; 17:1646-1656.

6. Bishop NA, Guarente L. Two neurons mediate diet-restrictioninduced longevity in C. elegans. Nature. 2007; 447:545-549.

7. Kaeberlein TL, Smith ED, Tsuchiya M, Welton KL, Thomas JH, et al. Lifespan extension in Caenorhabditis elegans by complete removal of food. Aging Cell. 2006; 5:487-494.

8. Lee GD, Wilson MA, Zhu M, Wolkow CA, de Cabo R, et al. Dietary deprivation extends lifespan in Caenorhabditis elegans. Aging Cell. 2006; 5:515-524.

9. Kapahi P, Zid BM, Harper T, Koslover D, Sapin V, et al. Regulation of lifespan in Drosophila by modulation of genes in the TOR signaling pathway. Curr Biol. 2004; 14:885-890.

10. Kaeberlein M, Powers RW, 3rd, Steffen KK, Westman EA, Hu $D$, et al. Regulation of yeast replicative life span by TOR and Sch9 in response to nutrients. Science. 2005; 310:1193-1196.

11. Vellai $T$, Takacs-Vellai $K$, Zhang $Y$, Kovacs AL, Orosz $L$, et al. Genetics: influence of TOR kinase on lifespan in C. elegans. Nature. 2003; 426:620.

12. Harrison DE, Strong R, Sharp ZD, Nelson JF, Astle CM, et al. Rapamycin fed late in life extends lifespan in genetically heterogeneous mice. Nature. 20009; 460:392-395.

13. Chen D, Thomas EL, Kapahi P. HIF-1 modulates dietary restriction-mediated lifespan extension via IRE-1 in Caenorhabditis elegans. PLoS Genet. 2009; 5:e1000486. 
